\title{
Observational Studies Are Complementary to Randomized Controlled Trials
}

\author{
Diana C. Grootendorst ${ }^{\mathrm{a}}$ Kitty J. Jager ${ }^{\mathrm{b}}$ Carmine Zoccali ${ }^{\mathrm{c}}$ Friedo W. Dekker $^{\mathrm{a}}$ \\ a Department of Clinical Epidemiology, Leiden University Medical Center, Leiden, and b ERA-EDTA Registry, \\ Department of Medical Informatics, Academic Medical Center, University of Amsterdam, Amsterdam, \\ The Netherlands; ${ }^{c}$ CNR-IBIM Clinical Epidemiology and Pathophysiology of Renal Diseases and Hypertension, \\ Renal and Transplantation Unit, Ospedali Riuniti, Reggio Calabria, Italy
}

\section{Key Words}

Observational studies $\cdot$ Randomized controlled trials . Chronic kidney disease $\cdot$ Treatment effect

\begin{abstract}
Randomized controlled trials (RCTs) are considered the gold standard study design to investigate the effect of health interventions, including treatment. However, in some situations, it may be unnecessary, inappropriate, impossible, or inadequate to perform an RCT. In these special situations, well-designed observational studies, including cohort and case-control studies, may provide an alternative to doing nothing in order to obtain estimates of treatment effect. It should be noted that such studies should be performed with caution and correctly. The aims of this review are (1) to explain why RCTs are considered the optimal study design to evaluate treatment effects, (2) to describe the situations in which an RCT is not possible and observational studies are an adequate alternative, and (3) to explain when randomization is not needed and can be approximated in observational studies. Examples from the nephrology literature are used for illustration.

Copyright $\odot 2009$ S. Karger AG, Basel
\end{abstract}

\section{Introduction}

A randomized controlled trial (RCT) is considered the gold standard study design to investigate the efficacy and effectiveness of drugs, medicines and interventions [1]. In the hierarchy of study designs to obtain evidence for treatment effect, RCTs and meta-analyses of RCTs are at the top and yield relatively the best evidence, while case reports are at the bottom (fig. 1). A meta-analysis can also be performed of observational studies, using the same methods as for RCTs, as has for instance been done by Lazarides et al. [2] in their meta-analysis of observational studies of dialysis access outcome in elderly patients with end-stage renal disease (ESRD). However, the evidence obtained from such a meta-analysis is less strong than that from a meta-analysis on RCTs. The hierarchy of study designs is used to translate the results of studies into clinical practice guidelines, such as the KDOQI, using the studies' levels of strength of evidence for rationale statements [3].

Even though RCTs are considered the best study design to examine treatment efficacy and effectiveness, such a study cannot always be conducted. There are 4 main reasons not to perform an RCT: (1) experimentation may be unnecessary, (2) inappropriate, (3) impossible, or (4) inadequate [4]. In these 4 situations, (also) well-de-

Diana C. Grootendorst

Department of Clinical Epidemiology C7-99

Leiden University Medical Center, PO Box 9600

NL-2300 RC Leiden (The Netherlands)

Tel. +31 71526 6914, Fax +31 71526 6994, E-Mail d.c.grootendorst@lumc.nl 


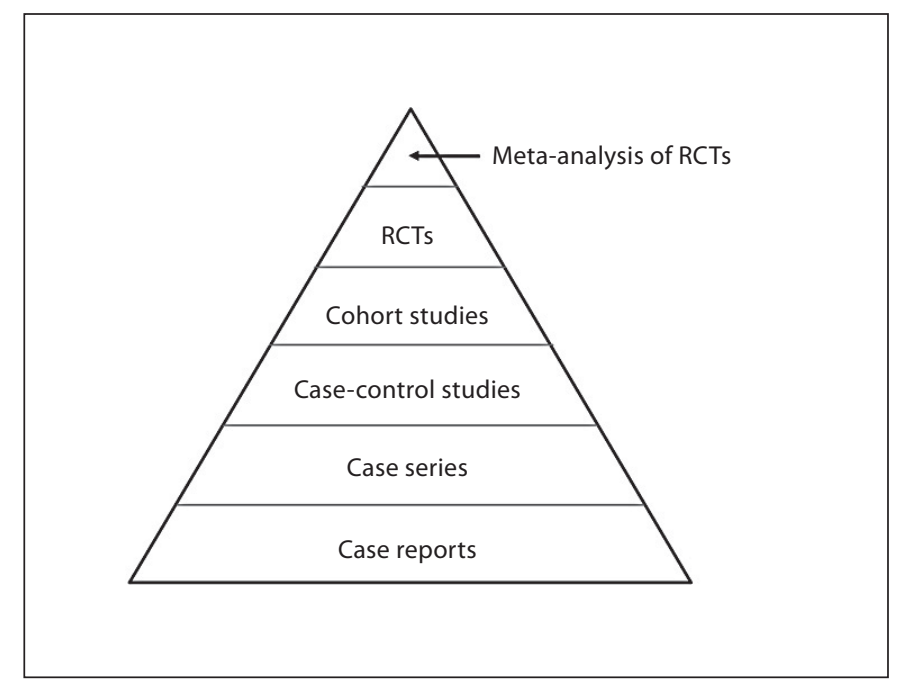

Fig. 1. Hierarchy of study designs to obtain evidence for treatment effect.

signed observational studies including cohort studies and case-control studies are used to demonstrate effectiveness of treatments, although for this specific purpose evidence is almost always less strong than in RCTs. Details concerning the design and conduct of cohort [5] and case-control studies [6] have been described in 2 separate manuscripts which appeared earlier in this series.

The aims of this review are (1) to explain why RCTs are considered the optimal study design to evaluate treatment effects, (2) to describe the situations in which an RCT is not possible and observational studies are an adequate alternative, and (3) to explain when randomization is not needed and can be approximated in observational studies.

\section{Advantages of RCTs to Evaluate an Intended Treatment Effect}

Randomization, concealment of treatment allocation and the possibility of double-blind administration of study medication are important key concepts of RCTs [1]. The random allocation of treatment avoids selection bias or confounding by indication, and is meant to create treatment groups that have comparable prognosis with respect to the outcome under study. Comparability of prognosis is important when investigating treatment efficacy and effectiveness as it is necessary to determine whether the observed treatment effect in the 2 groups is due to the intervention or due to the differences in prognosis at baseline.

With concealment of treatment allocation, i.e., the randomization and its code are not accessible to the treating physician, it is guaranteed that randomization is not influenced. This ensures the unprejudiced inclusion of patients in the study, which again contributes to comparability of the study groups. Finally, with double-blind administration of the study treatment (e.g. medication), the treatment effects can be recorded and reported in an objective manner by physician and patient, respectively.

Using an RCT to assess the intended effect of a treatment in comparison to another treatment, 2 different approaches can be used depending on the hypothesis and aim of the study. If one intends to demonstrate that a treatment is better than a reference treatment, a superiority trial is performed. In contrast, if the aim of the study is to demonstrate that 2 treatments have the same effect, an equivalence or non-inferiority trial is performed. These 2 types of trials have different methodological aspects and underlying assumptions, which are elegantly described by Christensen [7].

\section{Observational Studies instead of RCTs for the Evaluation of Intended Treatment Effects}

Some people examine the effect of a treatment in observational studies. Usually, a major drawback of such studies for the assessment of intended treatment effects is confounding by indication: those who receive the drug prescription generally differ from those who do not, according to the medical indication for which the drug was prescribed. Even if the comparison group represents patients with the same disease who received a different therapy or none at all, there will typically be differences in disease severity or other risk factors between populations who receive different treatments. These differences in general introduce a bias in the comparison [8]. In some cases, when an RCT is (1) unnecessary, (2) inappropriate, (3) impossible or (4) inadequate (table 1) [4], well-designed observational studies including cohort and casecontrol studies might provide estimates of treatment effect, provided the study is performed correctly. An important prerequisite in such observational studies is breaking the link between prescription and prognosis [9], for instance, by using an indicator variable that can be used to define the groups of patients to be compared and is independent from the prognosis of the patient. This can be seen as an approximation of randomization. Ex- 
Table 1. Situations in which RCTs cannot be conducted and observational studies are an alternative to doing nothing to estimate the effectiveness of treatment

\begin{tabular}{ll}
\hline $\begin{array}{l}\text { Criteria from } \\
\text { Black [4] }\end{array}$ & Specific reason \\
\hline RCT is unnecessary & $\begin{array}{l}\text { Treatment effect is so dramatic that un- } \\
\text { known confounding factors could be ig- } \\
\text { nored }\end{array}$ \\
\hline RCT is inappropriate & $\begin{array}{l}\text { When an outcome is rare, happens far in } \\
\text { the future and long follow-up is required } \\
\text { or when randomization could reduce ef- } \\
\text { fectiveness; mainly unintended treat- } \\
\text { ment effects }\end{array}$ \\
\hline RCT is not possible & $\begin{array}{l}\text { Clinician's refusal to participate, legal } \\
\text { obstacles and/or ethical considerations }\end{array}$ \\
\hline RCT is inadequate & $\begin{array}{l}\text { RCTs may have low external validity or } \\
\text { poor generalizability due to the extensive } \\
\text { inclusion and exclusion criteria for par- } \\
\text { ticipants }\end{array}$ \\
\hline
\end{tabular}

amples for each of these reasons are presented together with an explanation how the link between prescription and prognosis was broken, if applicable. It should be noted that in observational studies, just as in RCTs, one can choose to investigate superiority or equivalence of treatment using the same assumptions and methodology as in clinical trials [7].

\section{Observational Studies when It Is Unnecessary to Perform an RCT}

RCTs may be unnecessary to perform when the treatment effect is so dramatic that unknown confounding factors can be ignored [4]. A classic example is the introduction of hemodialysis in patients with ESRD. Up until the 1940s, patients with total renal failure usually died within 3 weeks. In 1943, Kolff [10] introduced the first dialyzer which prolonged life expectancy in patients with acute renal failure. In the 1960s, Scribner and colleagues added a Teflon shunt to enable dialysis treatment in chronic renal failure, which considerably prolonged the survival [11]. In this example, survival in a hypothetical control group not treated with chronic dialysis would be so poor, that a formal parallel-group randomized experiment would not be necessary. However, nowadays, the introduction of novel therapies or technologies is strictly regulated by federal authorities, which demand several RCTs demonstrating efficacy of the novel treatment.
Therefore, at present, it is difficult to imagine that novel compounds or technologies can be introduced for treatment based on observational data only.

\section{Observational Studies when It Is Inappropriate to Perform an RCT}

Adverse events or unintended treatment effects are outcomes that are usually rare (less than $\sim 1$ per 200 /year [9]) and may take quite some time to develop (longer than 1 year [9]). When studying adverse events or unintended treatment effects, observational studies are just as valid as and perhaps even more appropriate than RCTs, as allocation of treatment is usually not related to the prognosis with respect to this specific outcome or unintended effect [4].

An example of an observational study examining unintended treatment effects is the one by Kolesnyk et al. [12]. Angiotensin-converting enzyme (ACE) inhibitors and angiotensin II receptor blockers (ARBs) are frequently prescribed in peritoneal dialysis patients with the indication of control of hypertension or heart failure. Furthermore, angiotensin II is known to be a growth factor in the development of renal fibrosis [13]. Experimental animal studies have shown that ACE inhibitors have beneficial effects on the development of morphological peritoneal alterations by decreasing angiogenesis and fibrosis [14]. Kolesnyk et al. [12] were the first to hypothesize that long-term treatment with ACE inhibitors or ARBs might attenuate peritoneal alterations that can develop in longterm peritoneal dialysis patients. Performance of an RCT to investigate these unintended effects of ACE inhibitors and ARBs would be inappropriate since peritoneal alterations are relatively rare events which may develop over a relatively long time period.

\section{Observational Studies when It Is Not Possible to Perform an RCT}

In some circumstances, RCTs are hardly possible to perform, and researchers have to rely on observational studies for reasons including clinician's refusal to participate, legal obstacles and/or ethical considerations [4]. For example, in 1999, a study was published by Smets et al. [15], which reported on the comparison of the survival of patients with type 1 diabetes and ESRD who were transplanted with a kidney or simultaneously with pancreas and kidney. Prior studies had shown beneficial effects of a functioning pancreatic allograft on quality of life and secondary complications of diabetes $[16,17]$. However, initiation of RCTs so far had been thwarted by practical and ethical considerations [18]. In this case, the 
observational design as used by Smets et al. [15] was a valid alternative, since the allocation of treatment (type of transplant) was related to ZIP-code and not to prognosis.

\section{Observational Studies when RCTs Are Inadequate}

RCTs may have low external validity or poor generalizability due to the extensive inclusion and exclusion criteria for participants [4], which is illustrated for example in the study by Korevaar et al. [19]. The aim of this study was to compare the survival and quality of life between hemodialysis and peritoneal dialysis in an RCT among patients new on dialysis. Patients without medical, social or logistic objections against either hemodialysis or peritoneal dialysis were eligible for inclusion and randomization. An a priori sample size calculation showed that 50 patients per group would be needed. After an inclusion period of more than 3 years and screening of 1,232 patients, only 38 patients were enrolled in total. The trial was stopped prematurely due to disappointing enrollment rates. Of the patients screened, 459 did not meet the inclusion criteria, and 735 had a clear preference for either hemodialysis or peritoneal dialysis, showing a strong selection of participants. Interestingly, the results of the study showed that hemodialysis patients were at an increased mortality risk of approximately 3.6 compared to peritoneal dialysis patients during the first 4 years of dialysis. Due to the high selection of patients, these results may be difficult to generalize to all dialysis patients. An observational study investigating this issue would have the advantage of reflecting the actual prescribing of hemodialysis and peritoneal dialysis in health care, and the advantages of including broader patient populations also from non-teaching hospitals, all resulting in higher generalizability [20].

\section{Approximation of Randomization}

In some situations, the link between prescription of a treatment or intervention and prognosis can be broken. Randomization can then be approximated in observational studies. Strictly seen, an RCT would not be needed, and such observational studies may yield unbiased estimates of the treatment effect. In the earlier-mentioned study of Kolesnyk et al. [12], an observational follow-up study in dialysis patients who were treated by peritoneal dialysis for at least 2 years was performed in order to investigate the unintended treatment effects of ACE inhibitors and ARBs on peritoneal transport char- acteristics. ACE inhibitors and ARBs had been prescribed for blood pressure regulation and not for their possible membrane protective effects. The administration of ACE inhibitors and ARBs can therefore be considered a random process with respect to the prognosis of peritoneal membrane function, and the results can be expected to be valid.

As another example, an observational study which would compare the survival of kidney alone versus combined kidney-pancreas transplantation, selection bias or confounding by indication would be a major issue, since there is selection of patients for pancreas-kidney transplantation on good as well as poor health indicators. In the study by Smets et al. [15], approximation of randomization was elegantly established in their observational study. In The Netherlands, the renal replacement registry RENINE prospectively collects data from all patients with ESRD who need any form of renal replacement therapy. Furthermore, there is strict center allocation for kidney transplantation based on the place of residence of the patient. Finally, at the time of the study by Smets et al. [15], the vast majority of simultaneous pancreas-kidney transplantations were performed in Leiden, which is one of the 7 transplantation centers in The Netherlands. Together, this provided the opportunity to perform a population-based observational follow-up study on the effect of the inclusion of pancreas transplantation in a kidney transplantation program, free from selection bias. In the analysis, the survival of patients who were transplanted in Leiden (and thus of the majority receiving the combined transplantation) was compared to the survival of patients who were transplanted in the other regions (and mainly received kidney transplantation alone). It is unlikely that the prognosis of ESRD patients who live in the Leiden area was different from that of patients living in the non-Leiden area, and, therefore, this can be considered as a form of randomization.

Finally, randomization can be approximated in the investigation of interaction between genetic polymorphisms and treatment effect. Genetic polymorphism means the existence of 2 or more allele variants at the same locus within the DNA. Alleles are randomly assorted during gamete formation, independent from environmental factors [21]. Furthermore, therapy is prescribed to patients independent from knowledge about genetic polymorphisms. Thus, the interaction between genetic polymorphisms and treatment effect can validly be studied in observational studies. For instance, in a study by Donnelly et al. [22], the association of a single nucleotide polymorphism (rs17238540) in the 3-hydroxy-3-methylgluta- 
ryl coenzyme A reductase gene with lipid-lowering response to statins was studied in a large population-based cohort of patients with diabetes.

\section{Conclusion}

Although RCTs are the gold standard study design to estimate efficacy and effectiveness of treatment, well-designed observational studies can be an alternative to doing nothing when RCTs cannot be performed. Circumstances in which observational studies can or should replace RCTs for the estimation of treatment effect include: an RCT may be (1) unnecessary, (2) inappropriate, (3) im- possible, or (4) inadequate [4]. In these special circumstances, well-designed observational studies including cohort and case-control studies may provide estimates of treatment effect, provided the study is performed correctly and with extreme caution. It should be noted that the evidence obtained in observational studies is almost always less strong than in RCTs. Furthermore, caution and restraint should be used in the interpretation of treatment effect obtained from observational studies when the effects are small or inconsistent with other studies. Only when approximation of randomization can be obtained by breaking the link between prescription and prognosis, the obtained treatment effects in observational studies can be considered unbiased.

\section{References}

1 Stel VS, Zoccali C, Dekker FW, Jager KJ: The randomized controlled trial. Nephron Clin Pract 2009;113:c337-c342.

$\checkmark 2$ Lazarides MK, Georgiadis GS, Antoniou GA, Staramos DN: A meta-analysis of dialysis access outcome in elderly patients. J Vasc Surg 2007;45:420-426

$\checkmark 3$ National Kidney Foundation: K/DOQI clinical practice guidelines for chronic kidney disease: evaluation, classification, and stratification. Am J Kidney Dis 2002;39:S1S266.

4 Black N: Why we need observational studies to evaluate the effectiveness of health care. BMJ 1996;312:1215-1218.

$\checkmark 5$ Euser AM, Zoccali C, Jager KJ, Dekker FW: Cohort studies: prospective versus retrospective. Nephron Clin Pract 2009;113: c214-c217.

6 van Stralen KJ, Dekker FW, Zoccali C, Jager KJ: Case-control studies. Nephron Clin Pract 2009; 114:c1-c4.

7 Christensen E: Methodology of superiority vs. equivalence trials and non-inferiority trials. J Hepatol 2007;46:947-954.

8 Rothman KJ: Epidemiology. An Introduction. Oxford, Oxford University Press, 2002.

9 Vandenbroucke JP: When are observational studies as credible as randomised trials? Lancet 2004;363:1728-1731.
10 Kolff WJ: Lasker Clinical Medical Research Award. The artificial kidney and its effect on the development of other artificial organs. Nat Med 2002;8:1063-1065.

$\checkmark 11$ Scribner BH, Caner JE, Buri R, Quinton W: The technique of continuous hemodialysis. Trans Am Soc Artif Intern Organs 1960;6: 88-103.

12 Kolesnyk I, Dekker FW, Noordzij M, le Cessie S, Struijk DG, Krediet RT: Impact of ACE inhibitors and AII receptor blockers on peritoneal membrane transport characteristics in long-term peritoneal dialysis patients. Perit Dial Int 2007;27:446-453.

13 Wolf G, Neilson EG: Angiotensin II as a renal growth factor. J Am Soc Nephrol 1993;3: 1531-1540.

14 Duman S, Gunal AI, Sen S, Asci G, Ozkahya M, Terzioglu E, Akcicek F, Atabay G: Does enalapril prevent peritoneal fibrosis induced by hypertonic (3.86\%) peritoneal dialysis solution? Perit Dial Int 2001;21:219-224.

-15 Smets YF, Westendorp RG, van der Pijl JW, de Charro FT, Ringers J, de Fijter JW, Lemkes $\mathrm{HH}$ : Effect of simultaneous pancreas-kidney transplantation on mortality of patients with type-1 diabetes mellitus and end-stage renal failure. Lancet 1999;353:1915-1919.

16 Landgraf R: Impact of pancreas transplantation on diabetic secondary complications and quality of life. Diabetologia 1996;39: 1415-1424.
17 Luzi L: Pancreas transplantation and diabetic complications. N Engl J Med 1998;339: 115-117.

$>18$ Robertson RP, Holohan TV, Genuth S: Therapeutic controversy: pancreas transplantation for type I diabetes. J Clin Endocrinol Metab 1998;83:1868-1874.

$>19$ Korevaar JC, Feith GW, Dekker FW, van Manen JG, Boeschoten EW, Bossuyt PM, Krediet RT: Effect of starting with hemodialysis compared with peritoneal dialysis in patients new on dialysis treatment: a randomized controlled trial. Kidney Int 2003; 64:2222-2228.

20 Jager KJ, Stel VS, Wanner C, Zoccali C, Dekker FW: The valuable contribution of observational studies to nephrology. Kidney Int 2007;72:671-675.

21 Chmielewski M, Carrero JJ, Nordfors L, Lindholm B, Stenvinkel P: Lipid disorders in chronic kidney disease: reverse epidemiology and therapeutic approach. J Nephrol 2008;21:635-644.

22 Donnelly LA, Doney AS, Dannfald J, Whitley AL, Lang CC, Morris AD, Donnan PT, Palmer CN: A paucimorphic variant in the HMG-CoA reductase gene is associated with lipid-lowering response to statin treatment in diabetes: a GoDARTS study. Pharmacogenet Genomics 2008;18:1021-1026. 